\title{
Shakespeare et la France
}

\section{Gisèle Venet}

\section{(2) OpenEdition \\ Journals}

\section{Édition électronique}

URL : http://journals.openedition.org/shakespeare/641

DOI : 10.4000/shakespeare.641

ISSN : 2271-6424

\section{Éditeur}

Société Française Shakespeare

\section{Édition imprimée}

Date de publication : 1 novembre 2000

Pagination : 209-228

ISBN : 2-84269-407-4

\section{Référence électronique}

Gisèle Venet, "Shakespeare et la France », Actes des congrès de la Société française Shakespeare [En ligne], 18 | 2000, mis en ligne le 01 novembre 2007, consulté le 05 mai 2019. URL : http:// journals.openedition.org/shakespeare/641 ; DOI : 10.4000/shakespeare.641 


\section{S H A K E S P E A R E \\ \& L A F R A N C E}

Société Française Shakespeare

Actes du Congrès de 2000

* * *

Textes réunis et présentés par

Patricia DORVAL

publiés sous la direction de Jean-Marie MAGUIN 
Colloque honoré d'une subvention du Ministère de l'Éducation Nationale, de la Recherche et de la Technologie

Site Internet $:<$ http://alor.univ-montp3.fr/SFS/> Liste de diffusion : <sfs-1@smr1.univ-montp3.fr>

Tous droits de traduction, de reproduction et d'adaptation réservés pour tous les pays.

(C) 2000. Société Française Shakespeare, École Normale Supérieure, 45 rue d'Ulm, 75005 Paris.

ISBN 2-84269-407-4 


\section{S H A K E S PEARE ET LA F RANCE}

Shakespeare et la France ? Tout paraît inviter à s'intéresser à Shakespeare vu de France, terrain d'entente sans trop d'embûches puisque le génie shakespearien y est de plus en plus généreusement reconnu, et puisque les scènes françaises sont plus que jamais fascinées par l'audacieux défi que représente toujours pour un metteur en scène, un traducteur, un comédien la mise en spectacle d'un texte shakespearien. Le XVIIIe siècle, avec la naissance en Angleterre du «mythe» shakespearien et des grandes rééditions voire réécritures - des textes de Shakespeare, mais aussi de la fascination qu'exerce le modèle politique anglais sur l'Intelligentsia française pré-révolutionnaire aurait fourni l'occasion de retrouver leur cortège d'emprunts culturels et littéraires si salutaires à notre culture. Le phénomène le plus voyant de cette passation d'héritage reste sans doute encore la «lettre philosophique» sur la tragédie dans laquelle Voltaire propose sa traduction du monologue d'Hamlet dont, citant le vers en anglais «To be, or not to be, that is the question», il affirme qu'il «est su de tout le monde» ${ }^{1}$. Il en donne sa version dans la grande tradition de l'alexandrin français, rimé comme il se doit, qui aide à prendre la mesure de l'écart entre des styles tragiques dont l'un, l'anglais, appartient à l'apogée du théâtre baroque, à son économie de moyens pour des effets multiples, l'autre à un «classicisme» français dont il ne reste plus qu'un formalisme d'écriture au moment où Voltaire s'en saisit, bien qu'il rêve encore d'être le tragédien des Lumières : 
Demeure; il faut choisir et passer à l'instant

De la vie à la mort, ou de l'être au néant :

Dieux cruels! s'il en est, éclairez mon courage.

Faut-il vieillir courbé sous la main qui m'outrage,

Supporter ou finir mon malheur et mon sort?

Qui suis-je ? qui m'arrête? et qu'est-ce que la mort ? ${ }^{2}$

Il commente alors sa propre traduction, revendication d'une autre liberté : «Ne croyez pas que j'aie rendu ici l'anglais mot pour mot ; malheur aux faiseurs de traductions littérales, qui, en traduisant chaque parole, énervent le sens» ${ }^{3}$. Ce sens déjà «énervé», sans nerf et sans tension tragique, il l'a parfaitement identifié dans l'écriture profuse d'un Dryden, «auteur plus fécond que judicieux», et Voltaire en profite pour déplorer l'influence inverse, celle des Français sur les Anglais: "La coutume d'introduire de l'amour à tort et à travers dans les ouvrages dramatiques passa de Paris à Londres vers l'an 1600 avec nos rubans et nos perruques» ${ }^{4}$, identifiant de fait au passage, malgré la date trop précoce, les nouvelles inflexions du théâtre post-shakespearien, écriture tout autre, incomparable au sens premier du terme, sans implication péjorative. Et bien en prendrait aux critiques du théâtre anglais non shakespearien de s'en aviser aussi et de ne pas toujours chercher le sens et la portée de ces nouveaux auteurs - Fletcher, Webster, Chapman, Decker, Middleton, Massinger, Richard Brome, et bien d'autres - à la seule lumière du théâtre shakespearien, comme autant de formes dégradées ou d'imitations intempestives. Tout le théâtre baroque qui précède Dryden appartient encore à ce moment du génie anglais sur lequel Voltaire conclut sa dix-huitième lettre «Sur la tragédie»:

Il semble que les Anglais n'aient été faits jusqu'ici que pour produire des beautés irrégulières. Les monstres brillants de Shakespeare plaisent mille fois plus que la sagesse moderne. Le Génie poétique des Anglais ressemble jusqu'à présent à un arbre touffu planté par la nature, jetant au hasard mille rameaux et croissant avec inégalité et avec force; il meurt si vous voulez forcer sa nature et le tailler en arbre des jardins de Marly. ${ }^{5}$

Dans la longue histoire qui lie Shakespeare et l'imaginaire français, il aurait fallu rappeler ce vent, cette bourrasque de liberté 
que Shakespeare représente pour la génération romantique, longtemps soumise à la tyrannie de l'alexandrin, en même temps qu'aux régimes bourgeois post-révolutionnaires, et qui trouve dans ce génie anglais la matière d'un véritable «manifeste» politique autant que culturel. Pour Stendhal, dans son Racine et Shakespeare publié en 1823, l'échange fictif de lettres entre «un Classique» et «un Romantique» est l'occasion d'opposer deux modes d'écriture sous deux régimes politiques antinomiques et de comparer les contraintes imposées à Racine par l'oppression d'un régime despotique, l'absolutisme de Louis XIV, aux libertés dont jouit un Shakespeare sous le régime - quelque peu idéalisé d'émancipation politique qu'est à ses yeux le règne d'Élisabeth Ière.

Tard dans le XIXe siècle, c'est encore à Shakespeare que recourt Victor Hugo pour évoquer au nom de la génération romantique le génie tutélaire de la liberté, Shakespeare, «l'homme océan». Alors exilé politique à Jersey, il découvre en Angleterre et dans Shakespeare le refuge contre l'oppression anti-démocratique qu'est pour lui l'installation du régime d'Empire par. Napoléon III. II découvre dans le même temps les vertus créatrices d'une ascèse dans le vent d'hiver de l'exil. Ce sera la longue préface qui deviendra livre en 1864, Shakespeare, où Hugo fait état de ce rapport indissoluble entre exil et hiver, au cours d'une conversation avec son fils qu'il rapporte dans un moment qui scelle le lien entre Shakespeare et la France, avec le projet de transmettre en la traduisant l'intégralité d'une œuvre :

Dehors, il pleuvait, le vent soufflait, la maison était comme assourdie par ce grondement extérieur. Un père et un fils songeaient, absorbés peut-être par cette coïncidence d'un commencement d'hiver et d'un commencement d'exil.

Tout à coup, le fils éleva la voix et interrogea le père :

- Que penses-tu de cet exil ?

- Qu'il sera long.

- Comment comptes-tu le remplir?

Le père répondit :

- Je regarderai l'océan.

Il y eut un silence. Le père reprit :

- Et toi?

Moi, dit le fils, je traduirai Shakespeare. ${ }^{6}$ 
Curieusement, «cette coïncidence d'un commencement d'hiver et d'un commencement d'exil» pourrait bien dominer aussi la relation entre «l'homme océan» et la France, la France vue par Shakespeare, telle qu'il la met en scène au hasard de ses choix, comédies ou histoires, et parfois tragédies. Quelles images de la France s'attardent en effet dans l'imaginaire shakespearien? Quelles constantes ou quelle diversité éventuelle dans la perception de cette autre "puissante monarchie» (Henry $V$, Prologue) ${ }^{7}$ si longtemps impliquée dans l'histoire des rois anglais ? La France se réduit-elle à un simple territoire à reconquérir qui aurait vu les prestigieux faits d'armes du Prince Noir à Crécy ou à Poitiers, d'Henry V à Azincourt, mais aurait aussi incarné la perte de toutes les valeurs anglaises dès lors que le royaume de France retrouvait son autonomie lorsque survint la guerre des Deux Roses ? C'est bien sûr dans les pièces historiques que la présence de la France est la plus évidente, présence d'une France quasi réelle dans cet imaginaire et utilisée à des fins épiques, éthiques et politiques. D'autres images cependant, plus diffuses, plus discrètes, moins annexées à la seule réalité historique, renvoient aussi à une France imaginaire : dans des comédies comme Love's Labours Lost, on voit une Princesse de France venir plaider la cause de son père auprès du roi de Navarre, ou dans All's Well That Ends Well, une jeune orpheline guérir un roi de France, et la France apparaît alors comme lieu des renversements du sentiment amoureux mais aussi lieu de deuil ou de frustration, d'où il faut aussi s'exiler ou revenir sans amour. Dans As You Like It, la France, si tant est que la Forêt d'Ardennes y soit située, est à la fois terre d'exil et d'accueil, liberté arcadienne mais aussi pastorale d'hiver, avec ce souffle glacial du vent - «churlish chiding of the winter's wind» (II.1.7).

Est-ce une gémellité ou une altérité radicale avec l'Angleterre qu'exprime alors dans ces pièces le pays imaginaire ou le pays imaginé qu'est la France? Faut-il voir en ces deux pays deux espaces conjoints comme l'image d'héraldique le suggère dans Edward III ${ }^{8}$ - «The arms of England and of France unite / Are quartered equally by herald's art» (III.1.73-5) -, ou des lieux aux qualités et dimensions antithétiques ? Pourquoi cette référence à un ailleurs si proche et si radicalement différent, face à une Angleterre volontiers mythifiée, glorifiée?

L'Angleterre mythique, c'est celle qu'évoque ou plutôt invoque un John of Gaunt désespérant de l'avenir dans Richard II ${ }^{9}$ et idéalisant ce que fut, ce qu'il souhaite que soit toujours l'Angleterre, 
la nation joyau, «This precious stone set in a silver sea» (II.1.46). Idéalisée, l'Angleterre l'est comme paradigme de la perte toujours menaçante, implicite dans toute référence au paradis, ici «this other Eden, demi-paradise» (II.1.42). Lieu clos, naturellement protégé tant qu'il reste clos «against infection and the hand of war» (II.1.44), l'Angleterre est ceinte d'une clôture circulaire, doublement matérialisée comme protectrice, la mer ayant «the office of a wall» ou, plus explicite encore d'un imaginaire de l'encerclement défensif : «a moat defensive to a house» (II.1.48). La référence à «house» ici, même si elle peut renvoyer métaphoriquement au vaste domaine idéal, la «Country House», accentue encore la réduction de l'échelle de grandeur et l'impression de confinement. Dans l'imaginaire de Gaunt, l'utilisation de «blessed spot» implique encore et toujours le jardin clos, comme le «teeming womb of royal kings» (II.1.51) implique le ventre rond, image d'une sphéricité symbolique. L'imaginaire shakespearien de la nostalgie idéalise volontiers le monde parfait en sphère close sur sa propre finitude : lorsqu'Othello, avec la fin de son amour pour Desdémone, fait l'expérience du désastre, au sens propre de chute des astres hors de leurs sphères - «Methinks it should be now a huge eclipse / Of sun and moon, and that th'affrighted globe / Should yawn at alteration» (V.2.100-2) - , il se prend à rêver au monde parfait qui lui serait redonné - «Of one entire and perfect chrysolite» (V.2.144). Ce même recours à l'imaginaire de la sphère protectrice ou idéale, du repliement nostalgique sur un monde clos, on le retrouve jusque dans un roman contemporain de nous, 1984, de George Orwell. Au moment où, dans le roman, Wilson croit retrouver la possibilité d'un amour vrai, dans une clairière symbolique qui a la fonction d'une pastorale nostalgique, il redécouvre dans le même temps une sphère, équivalente dans son fonctionnement de la chrysolite d'Othello: dans une boutique de brocanteur, envahie d'objets venus du passé, il achète un presse-papier, une sphère de verre transparente qu'il peut retenir dans sa main, venue d'un temps antérieur à la catastrophe qui a instauré, avec le règne de «Big Brother», un temps sans mémoire individuelle.

L'idéalisation du parfait joyau qu'est l'Angleterre dans son monde clos s'accompagne chez Gaunt de la lamentation qui contient dans sa concision l'histoire des relations entre la France et l'Angleterre : «That England that was wont to conquer others, / Hath made a shameful conquest of herself» (Richard II, II.1.65-6). L'histoire d'un passé glorieux, aux conquêtes répétées - «was 
wont» - doit faire face au pitoyable devenir, un devenir qui se ferme, figé dans le present perfect «hath made». Le dernier vers fait référence à la perte de tous les territoires de France telle que Shakespeare la met en scène dans la première partie d'Henry $V I$, où cette perte est moins due à la vaillance des Français au siège d'Orléans qu'à leur perfidie ou aux faux miracles d'une sorcière, Jeanne d'Arc, osant se faire passer pour descendante de rois (l Henry VI, V.4.38) ; ou encore due aux divisions politiques internes dans le camp anglais, qu'il s'agisse des machinations de Suffolk pour que Henry VI épouse Marguerite d'Anjou dont lui-même est amoureux, ou qu'apparaisse au grand jour la volonté de puissance du Lord Protector Gloucester. La vaillance anglaise sur les champs de bataille, quant à elle, reste intacte, comme en témoigne la mort de Talbot qui succombe à la seule traîtrise après qu'il a reçu entre ses bras son fils héroïque, lui aussi mort au combat (I Henry VI, 5-6-7). Cette vaillance, une réplique encore en témoigne, celle de Lucy au Dauphin Louis qui demande : «On what submissive message are you sent ?» (IV.7.53), et s'entend répondre : «Submission, Dauphin!'Tis a mere French word» (54). L' «infection» tant redoutée par Gaunt a bel et bien pénétré de l'extérieur le camp anglais. Tout au long de 2 Henry VI et 3 Henry VI, le mariage du trop jeune roi avec Marguerite d'Anjou apparaît comme l'erreur initiale, l'introduction du corps étranger qui provoque et maintient la scission entre les deux Roses : «O blood-spotted Neapolitan / Outcast of Naples, England's bloody scourge...» (2 Henry VI, V.1.117-8) - par quoi Margaret, fille du roi René de Naples et d'Anjou est désignée.

Les conquêtes heureuses évoquées par Gaunt occupent deux pièces, «épiques» chacune à sa manière, Edward III et Henry $V$, dont les parentés tiennent aux épisodes historiques mis en scène mais aussi à des structures dramatiques et à des thématiques qui se répètent de l'une à l'autre. Les conquêtes qu'on y retrouve ont toutes pour visée la reconquête du territoire perdu, la France, décrite comme élargissement de l'espace dans Henry $V:$ :[to rule] in large and ample empery / O'er France and all her almost kingly dukedoms» (I.2.226-7); dans Edward III, le Prince Noir songe à l'exemple que seront ses exploits pour de nouvelles générations qui «Might thereby be inflamed with such resolve / As not the territories of France alone, / But likewise Spain, Turkey and what countries else / That justly would provoke fair England's ire / Might at their presence tremble and retire» (Edward III, V.1.231-4). Dans les deux cas, l'aventure épique sur la terre de France commence en effet par 
la revendication d'un espace au regard duquel le seul territoire de l'Angleterre est celui d'un confinement, menacé de nouvelles amputations de son intégrité : dans Henry $V$, le roi prépare son départ vers la France mais «the weasel Scot» (I.2.170) doit d'abord être contenu; Canterbury a lui aussi recours à l'image de la muraille : «They of those marches» - faisant référence aux baronnies du Nord - «Shall be a wall sufficient to defend / Our England» (I.2.140-2). Le danger toujours présent d'une pénétration de l'espace clos s'exprime dans l'image de la brèche employée dans ce contexte par Henry : «For you shall read that my great-grand father / Never went with his forces into France / But for the Scot [...] came pouring like a tide into the breach», menace de déferlement dangereux comme la mer, mais aussi d'encerclement : «girding with grievous sieges castles and towns» (152). Ce «great-grand father», Édouard III, dans la pièce éponyme, doit faire lever un véritable siège avant de pouvoir partir pour la France : «now the tyrant [le roi David d'Écosse] hath begirt with siege / The Castle of Roxborough» (Edward III, I.1.129-30). Les Écossais, «the stealing foxes» (I.2.90) dans cette pièce, se dispersent sans coup férir, mais un autre siège s'impose à Édouard III, l'amour illicite qu'il éprouve, au premier regard, pour l'occupante du château assiégé et délivré, la comtesse de Salisbury, mariée, qui avait failli être violée par les Écossais et se retrouve aux prises avec le désir impétueux et impérieux d'un roi. Une parenté implicite à nouveau s'esquisse avec Henry $V$, un obstacle moral à la reconquête de la France doit à nouveau être levé, comme si dans cet espace clos de l'Angleterre un avilissement du désir menaçait, avilissement qu'il faut aussi contenir avant cette reconquête de la France qui dès lors apparaît comme une quête sacralisée, méritée, et qui requiert de ses rois une ascèse morale avant de s'y risquer. Ainsi une jeunesse aventureuse avait paru compromettre l'avenir du Prince Hal comme roi ; et pourtant, comme l'observe Canterbury, «The courses of his youth promised it not / The breath no sooner left his father's body / But that his wildness mortified in him / Seemed to die too» (I.1.25-8). La mise à l'épreuve d'Henry $\mathrm{V}$ se concrétisera dans le choix contraignant tout au long de la pièce qui lui impose de ne pas grâcier ses anciens compagnons de débauche : Falstaff meurt le cœur brisé de chagrin, Bardolph est pendu pour un ciboire. Édouard III, quant à lui, est soumis dans son âge mûr au désir illicite de la femme parfaite à ses yeux, mais mariée et fidèle, qu'est la comtesse de Salisbury, et une dramatisation de la tentation occupe tout l'acte II, l'acte, soit dit en 
passant, que la critique textuelle attribue à Shakespeare. Même après avoir renoncé à l'acte impur - «graceless lust» (Edward III, II.1.430) - en retrouvant dans les traits de son fils, le Prince Noir, ceux de son épouse, il menace de succomber à nouveau devant le sourire de la Comtesse venue prendre congé : «Why, there it goes. That very smile of hers / Hath ransomed captive France» (Edward III, II.103-4). Il semble qu'une même ascèse soit requise de ce roi comme elle le sera d'Henry V : «Give me an armour of eternal steel, / I go to conquer kings ; and shall I not then / Subdue my self and be my enemies' friend ?» (II.2.96-8). Une même sacralisation de la mission guerrière y est symbolisée par la sacralité de l'adoubement de son fils, le Prince Noir, fait chevalier après la bataille de Crécy (Edward III, III.3.172-6), bataille qui avait ellemême valeur de rite de passage pour le jeune chevalier. La reconquête fonctionne comme un roman de chevalerie, avec sa mythique et sa mystique, et la pièce Edward III vient heureusement corroborer la mythique d'Henry $V$, pièce trop souvent réduite, vue de notre siècle, à une simple épopée ironique et qui pourtant fonctionne comme rite de passage, comme rite expiatoire aussi avec la mort des compagnons, la traîtrise de l'ami, Lord Grey, le poids de la responsabilité du père dans la mort de Richard II.

Épique et éthique, la reconquête n'en est pas moins politique : dans les deux pièces, à nouveau, s'impose d'entrée de jeu l'exposé des droits à la couronne de France dont les rois anglais furent spoliés et la mise en avant par les Français de la loi salique, abusivement appliquée pour écarter les rois anglais descendant par les femmes, en l'espèce la mère d'Édouard III, Isabelle de France, sœur aînée du Valois régnant. Si ironique que soit l'exposé de Canterbury dans Henry V(I.2.33-95), le titre de roi de France est revendiqué comme de droit: «Usurped from you and your progenitors» (I.2.95). De même dans Edward III, l'arrogant envoyé français, Lorraine, s'entend répondre : «Tell him the crown that he usurps is mine» (I.1.80), après une présentation de la loi salique non moins ironiquement placée dans la bouche d'un Français transfuge, le comte d'Artois, venu chercher refuge et faire allégeance auprès d'Édouard III. Un même fonctionnement se retrouve dans les deux

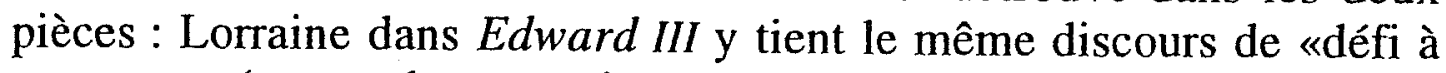
outrance» (terme de tournoi) que le Dauphin lors de son présent insolent des balles de tennis dans Henry $V$ (défi à soutenir un tournoi bouffon, tel qu'il s'en tenait en période de mi-carême, lancé à un prince bouffon ${ }^{10}$, «mad cap prince»). De même, selon le schéma 
désormais familier depuis Marlowe (dans Tamerlan, l'adversaire du conquérant a à peine le temps de formuler ses vantardises qu'il est écrasé sans appel, vaincu parfois d'un regard ou d'un mot - «your words are swords»), l'arrogance française est d'autant plus grande qu'elle est suivie plus vite d'un écrasement total. Dans Edward III, Charles de Normandie, alors Dauphin, a tout juste le temps de voir les Anglais comme autant de «Bloodthirsty and seditious Catilines» (III.1.14), ou comme autant de «ever-bibbing epicures» (26); de même son père, le roi Jean trouve à peine celui d'ironiser - "There comes a hare-brained nation, decked in pride» (51) - ou de se vanter - «At sea we are as puissant as the force / Of Agamemnon in the haven of Troy» (54-5) - ; ou encore le jeune fils Philippe de s'apprêter à donner la chasse - «Chase those straggling upstarts» (112) - que la bataille est livrée et perdue par ces mêmes Français : «We have untimely lost and they have won» (184). Cette imprévision, un bourgeois français la commente ironiquement par la fable de la Cigale et de la fourmi (III.2.14-26), ou plus tard le Dauphin Charles lui-même en tire une leçon bien tardive : "This day has set derision on the French / That all the world will blurt and scorn at us» (IV.6.44-6).

Le même effet de contraste ironique montre dans Henry $V$ la légèreté d'un autre Dauphin de France vantant son cheval à la veille d'Azincourt tandis que le roi d'Angleterre rend une visite nocturne à ses troupes; ou encore oppose le sérieux des futurs vainqueurs, les Anglais préoccupés du lendemain, aux plaisanteries du Connétable de France qui les décrit comme «yon poor and starved band», inquiet seulement de manquer de besogne sur le champ de bataille : «There is not work enough for all our hands, / Scarce blood enough in all their sickly veins / To give each naked curtle-axe a stain / That our French gallants shall today draw out / And sheathe for lack of sport» (IV.2.19-22).

Dans les deux pièces, un même transfert cynique des responsabilités sur l'adversaire fait de la victime des horreurs de la guerre son propre bourreau, exonérant du même coup le grand ancêtre Édouard III comme le jeune roi Henry V de la responsabilité morale des morts et des tortures subies, comme Tamerlan transférait sur les assiégés de Damas la responsabilité des meurtres et des viols qui devaient suivre la prise de la ville. Édouard III fait reproche au roi Jean de sa résistance à son avancée sur le territoire français : 
But had you done at first as now you do, How many civil towns had stood untouched That now are turned to ragged heaps of stones? How many people's lives might'st thou have saved That are untimely sunk into their graves?

(Edward III, V.1.202-6)

De même, Henry $\mathrm{V}$ devant Harfleur menace de surpasser Hérode en cruauté si les portes de la ville ne sont pas ouvertes par le Gouverneur: "This is the latest parle we will admit / [...] Therefore you men of Harfleur / Take pity of your town and of your people» (III.4.27-8). Il semble bien que l'épopée au passé, la nostalgie des espaces offerts à la conquête ramène ainsi l'imaginaire des anciens jours, l'imaginaire d'un espace ouvert, espace de circulation, de chevauchée, espace de devenir à opposer à l'imaginaire du lieu clos, idéalisé mais oppressant de circularité réduite, enclose. Imaginaire d'ouverture, de devenir, dont le prix à payer est la guerre, imaginaire d'ouverture dont le prix est aussi souvent l'exil et l'hiver.

Les nouvelles de France dans King Lear sont des nouvelles d'exil même si pour Cordelia, comme pour Kent, «Freedom lives hence, and banishment is here» (I.1.80). L'adoption de Cordelia par France se fait au prix d'un rejet de tous les liens intimes jusqu'ici parfaits avec le père qui maintenant la bannit : «Unfriended, newadopted to our hate / Dower'd with our curse and stranger'd with our oath» (I.1.202-3). La guerre à nouveau y occupe une fonction ambivalente de restauration: c'est de France que viennent les armées de Cordelia, non pas armées ordinaires, de conquête - «No blown ambition doth our arms incite» (IV.4.27) - mais de reconquête de la véritable intégrité du territoire perdu, celui de l'amour du père, bientôt restreint au cercle clos d'une prison. Dans Richard II, parcours inverse, la reine Isabelle pense trouver refuge en France, mais pour ce qui ne sera plus, sans Richard, qu'un long hiver d'exil.

Une autre France, non historique, et à peine géographique pourrait-on dire, s'esquisse dans le monde des comédies, pour offrir encore un espace ambivalent où des mythologies se répètent, se retournent, se défont ; où l'exil, intérieur ou spatial, a sa part, dans un climat d'hiver ou d'hiver affectif, faisant de ces comédies situées en France autant de contes d'hiver et d'exil. 
As You Like It est à ce titre exemplaire. La forêt d'Ardennes y est empruntée à la littérature plus qu'à la géographie, au roman de Lodge, Euphues Golden Legacie, plus qu'à la France réelle, pour y devenir le lieu d'une pastorale moralisée mais aussi d'une déstabilisation, voire d'un renversement de toutes les conventions de l'amour pétrarquiste. Dans cette forêt, «More free of peril than the envious court» (II.1.4), un duc mythique, banni de sa cour, y célèbre «les bienfaits de l'adversité» (II.1.12), redécouvrant un Éden paradoxal, d'après la chute, un «semi-paradise» hivernal où se perçoit pour le meilleur «the penalty of Adams, the seasons' difference», la fin de cet âge d'or sans saisons que fut l'état paradisiaque. L'exil est à nouveau la rencontre avec l'hiver, un hiver didactique, qui instruit sur le vrai sens de l'être : «Even till I shrink with cold, I smile and say: / 'This is no flattery: these are counsellors / That feelingly persuade me what I am'» (II.1.9-11). Un autre duc, «co-mate and brother in exile», et répondant au nom français d'Amiens, connote le thème de la France sans pour autant chanter les vignobles et la douceur d'y bien vivre. Au contraire, ce ne sont que références à l'hiver, même pour chanter la pastourelle «Under the greenwood tree». Le refrain en est plusieurs fois repris : «Here shall he see / No enemy / But winter and rough weather» (II.5.33-40), chant de l'amical hiver qui s'amplifie à la mesure d'une strophe entière : «Blow, blow, thou winter wind / Thou art not so unkind / As man's ingratitude, / Thy tooth is not so keen / Because thou art not seen / Although thy breath be rude...» (II.7.172-88). Rosalinde elle-même, malgré la liberté paradoxale qu'offre l'exil, comme pour Kent - «Now go we in content / To liberty and not to banishment» (I.3.132-3) —, s'arrête au seuil de la forêt pour dire sa perplexité : «Well, this is the forest of Arden»(II.4.11), tandis qu'Orlando, décrivant ce même lieu comme «desert inaccessible» (II.7.109), rêve d'un retour à l'anti-pastorale, à la vie civilisée d'avant l'exil: "If ever you have looked on better days, / If ever been where bells have knolled to church, / If ever sat at any good man's feast...» (II.7.110-5). Cet idéal, le duc exilé le répète mot pour mot tant il y retrouve sa propre nostalgie. Rosalinde dira elle aussi tout de go son désir de rentrer - formulation ambiguë du retour qui suit la découverte du mouchoir ensanglanté d'Orlando : «I would I were at home» (IV.3.159). Ambivalence dès lors que cette liberté d'un exil vécu comme une mise à l'épreuve, sur cette terre de France où viennent aussi se perdre tous les réflexes acquis. Les jeux amoureux de la pastorale hérités des sonnettistes, ce sonnet même 
sur lequel Orlando revient en scène après avoir choisi l'exil, n'auront plus cours devant la surprenante Rosalinde travestie en garçon, déguisement de carnaval, de «midsummer madness» comme dans Twelfth Night, mais pour une nuit de mai qui, dans ce monde hivernal, tarde à venir. Même la rencontre d'amants qui ne connaissent pas de saisons, les amants de la pastorale littéraire, Phoebe et Silvius, n'y changera rien, tant la dérision à laquelle les soumet Shakespeare nie tout épanouissement à venir. Prise à contrepied, ou plutôt au pied de la lettre, la pastorale retrouve ses racines rustiques avec les mains d'Audrey empestant l'odeur d'étable, ou ses origines mythiques avec la face de lune prêtée à Phoebe, dérision des topoi poétiques par quoi la pastorale ici se fait «satyre» comme on écrivait alors le mot satire, caprice de bouc plus que poème ovidien, ce qui selon Touchstone est tout un. Conte d'exil et d'hiver, As You Like It ouvrait sur un espace de découverte et de liberté mais ce n'était donc que pour y rencontrer à nouveau la nécessité d'une ascèse, ascèse sérieuse du duc et de sa cour bannie, ascèse joueuse imposée à Orlando par une Rosalinde meurtrie à son propre jeu.

Shakespeare n'en finit pas d'ouvrir l'espace vers une France qui renvoie à une frustration imposée comme nécessaire et referme le devenir sur une éthique, si rieuse ou moqueuse qu'elle soit. La même humeur se jouait du thème de l'ascèse imprévue, dans Love's Labours Lost, où sur un territoire, la Navarre, se présentait une princesse de France ; et une même dérision des traditions littéraires, de l'écriture des sonnets et de la poésie amoureuse codifiée en résultait. Un exil volontaire, la retraite studieuse du roi de Navarre et des aristocrates qui l'accompagnent, «co-mates in exile» à leur façon, y fonctionne comme un conte d'hiver à nouveau dont le masque des «Moscovites» venus du froid donne une moqueuse version. Mais alors même que la princesse et ses narquoises consœurs s'apprêtent peut-être à céder aux avances de Navarre et des siens, enfin démasqués dans leur contradiction entre retraite studieuse et poursuite amoureuse, un deuil final, la mort du roi de France, survient comme privation ajoutée aux jeux de l'amour verbal, autant de «peines d'amour perdues», de conventions en vain subies, pour rejeter un dénouement heureux loin par-delà le temps du dénouement de la comédie, là où nul n'a accès. Comme si Shakespeare à nouveau, par le jeu des imaginaires, se privait luimême de ce qu'il manie si bien, les conventions littéraires, trouvant dans cette privation sa jubilation supplémentaire de poète baroque, 
faisant sa joie perverse de toutes les mutilations culturelles qu'il s'impose.

Un dernier jeu pervers avec les conventions de l'amour courtois se révèle plus dévastateur dans une autre «comédie française», All's Well That Ends Well, commencée avec le deuil des pères, et reposant sur une subversion radicale de la cour d'amour pétrarquiste. Malgré la beauté des terres en Roussillon qui fera rêver Bertrand dans son exil italien, c'est un domaine stérile que Shakespeare met en scène : un exil intérieur plus grave que tout exil d'hiver affecte Hélène, puisque le jeune Bertrand quitte les lieux, et pour la fuir. La mort des pères qui libère les amants dans d'autres comédies pour les jeux de l'amour, ici se fait empêchement à aimer. L'épisode du roi de France mourant, guéri par la jeune Hélène fille d'un célèbre médecin, est à l'origine d'une structure de conte de fées: Hélène reçoit en récompense selon son vœu la main de Bertrand. Au seuil de cette cour d'amour à l'envers où elle choisit parmi les jeunes courtisans et n'est jamais choisie (II.3), Hélène se découvre l'antiDiane de la tradition amoureuse : "Now, Dian, from thy altar do I fly, / And to imperial love, that god most high, / Do my sighs stream» (II.3.73-5). Désormais figure paradoxale de la subversion du mythe de Diane, contrainte à la chasteté qui lui pèse (elle en fait confidence au bouffon Paroles), elle devient chasseresse de l'amant qui la fuit et ne fait rien pour la contempler nue. Victime de Vénus à sa proie attachée, elle le suivra dans son exil, un exil au cours duquel lui, Bertrand, rencontre enfin sa Diane, une belle florentine, qui selon les conventions amoureuses se refuse à lui tandis qu'il sonnettise. Car Bertrand n'a vu dans le conte de fées que son envers, un cauchemar, et marié avant d'avoir fait ses armes lors d'une cour d'amour, il prend les armes et s'enfuit à Florence. C'est là que, sous couvert de saint pèlerinage, Hélène, par un subterfuge scabreux, prend au lit la place d'une chaste Diane que rêvait de déflorer Bertrand et devient enfin la Vénus légitimée par le mariage antérieur à tout amour. Comédie du déni d'amour, All's Well That Ends Well contrevient à toutes les conventions littéraires du pétrarquisme, ici encore subverties par le simple recours à la littéralité, au refus des sens mythiques et des poétiques métaphoriques. Sur cette terre de France, une Hélène sans la beauté ni l'attrait qu'aurait dû lui valoir son nom est devenue la figure hybride d'une nouvelle sensibilité vénéneuse, la Diane-Vénus de l'érotisme baroque. 
Peut-on se dire qu'au fil de cet itinéraire imaginaire qui suggère des liens entre Shakespeare et la France, des constantes se dégagent au milieu de la multiplicité des contrastes ? Qu'une sorte d'ascèse paradoxale apparaît, ascèse de poète bien sûr avec ce goût de subvertir des pratiques, des structures, des conventions dont il a la parfaite maîtrise, bourreau de lui-même pour une jubilation supplémentaire, celle que produit l'exploration d'une nouvelle sensibilité qui s'affirme, en déséquilibre, entre espaces clos et espaces ouverts, cultures closes de la répétition et structures ouvertes de la subversion, la sensibilité baroque?

Hugo ne nomme pas le baroque mais le pressent lorsqu'en 1864, l'année même qui voit paraître la description de la littérature baroque par Burkhaert, il écrit sa longue préface en forme de livre aux traductions de son fils François-Victor. Au seuil de son étude, de la terre d'exil où il se trouve, l'Angleterre à laquelle il dédie son livre, «glorification de son poète», et que, «comme terre illustre et libre», il admire «et comme asile» il aime, Hugo décrit l'homme océan avec les mots qu'il faudrait pour décrire l'œuvre océan, l'œuvre baroque: "cette âcreté utile, cette amertume qui fait l'assainissement de l'univers, cet âpre sel sans lequel tout pourrirait ; ces colères et ces apaisements, ce tout dans un, cet inattendu dans l'immuable, ce vaste prodige de la monotonie inépuisablement variée, ce niveau après ce bouleversement, ces enfers et ces paradis de l'immensité éternellement émue, cet infini, cet insondable..., vous avez Eschyle, vous avez Isaïe, vous avez Juvénal, vous avez Dante, vous avez Michel-Ange, vous avez Shakespeare, et c'est la même chose de regarder ces âmes et de regarder l'Océan» ${ }^{11}$.

Gisèle VENET

Université Paris III - Sorbonne Nouvelle

NOT E S

${ }^{1}$ Voltaire, Mélanges, éd. J. Van den Heuvel, Gallimard, «Bibliothèque 
de la Pléiade», 1961, p. 82-3.

${ }^{2}$ Ibid., p. 82-3.

3 Ibid., p. 83.

${ }^{4}$ Ibid., p. 84.

5 Ibid., p. 84.

${ }^{6}$ Victor Hugo, Shakespeare, Paris, Librairie Internationale, 1864, p. XIII-XIV.

${ }^{7}$ Henry $V$, édition bilingue de Gisèle Venet, traduction de Jean-Michel Déprats, Folio Théâtre, Gallimard, 1999.

${ }^{8}$ King Edward III, éd. Giorgio Melchiori, Cambridge University Press, 1998.

${ }^{9}$ Richard II, édition critique de Margaret Jones-Davies, traduction de Jean-Michel Déprats, Folio Théâtre, Gallimard, 1998.

${ }^{10}$ Voir Gisèle Venet, préface à Henry V, op. cit., p. 25-32.

11 Shakespeare, op. cit., p. XVI. 